\title{
Surgery for infective endocarditis complicated by cerebral embolism: A consecutive series of 375 patients
}

\author{
Martin Misfeld, MD, PhD, Felix Girrbach, MD, Christian D. Etz, MD, PhD, Christian Binner, MD, \\ Konstantin V. Aspern, MD, Pascal M. Dohmen, MD, PhD, Piroze Davierwala, MD, \\ Bettina Pfannmueller, MD, PhD, Michael A. Borger, MD, PhD, and Friedrich-Wilhelm Mohr, MD, PhD
}

Objective: To determine the influence of silent and symptomatic cerebral embolism on outcome of urgent/emergent surgery after acute infective endocarditis (AIE).

Methods: From a total of 1571 patients with AIE admitted to our institution between May 1995 and March 2012 about one-quarter (375 patients; mean age, $61.8 \pm 13.6$ years) presented with cerebral embolism confirmed by cranial computed tomography. Isolated aortic valve endocarditis was present in 165 patients (44\%), 132 patients $(36 \%)$ had isolated AIE of the mitral valve, and $64(17 \%)$ patients had left-sided double valve endocarditis.

\begin{abstract}
Results: Although the majority of patients presented with neurologic symptoms, 1 out of 3 patients experienced a so-called silent asymptomatic cerebral embolism or transient ischemic attack $(\mathrm{n}=135)$. The rate of silent embolism was equivalent in patients with isolated aortic valve versus isolated mitral valve endocarditis (37\% vs $34 \% ; P=.54)$. Comparing patients with silent embolism versus symptomatic embolism, 18 patients with silent embolism versus 12 patients with symptomatic embolism developed postoperative hemiparesis $(P=.69)$. Three versus 4 had severe postoperative intracerebral bleeding $(P=.71)$. Median follow-up of survivors with cerebral embolism was 4.1 years (935 cumulative patient-years). Hospital mortality was $21.4 \%$ versus $19.6 \%(P=.68)$, with a long-term survival of $45 \% \pm 5 \%$ versus $47 \% \pm 4 \%$ at 5 years $(P=.83)$ and $40 \% \pm 6 \%$ versus $32 \% \pm$ $5 \%$ at 10 years $(P=.86)$. Independent risk factors of mortality were age at surgery $(P<.01)$, chronic obstructive pulmonary disease $(P=.01)$, preoperative requirement of catecholamines $(P=.02)$, dialysis $(P<.01)$, and duration of cardiopulmonary bypass $(P<.01)$.

Conclusions: Survival after surgery for AIE is significantly impaired once cerebral embolism has occurred; however, it does not differ in patients with symptomatic versus silent cerebral embolism. Routine computed tomography scans are therefore mandatory due to the high incidence of asymptomatic cerebrovascular embolism-which appears to be equally as dangerous as symptomatic embolism. (J Thorac Cardiovasc Surg 2014;147:1837-46)
\end{abstract}

Cerebrovascular embolic events in the setting of acute infective endocarditis (AIE) are frequent and have been proposed to impair short- and long-term survival. ${ }^{1,2}$ In particular, hemorrhagic transformation of septic embolic cerebral lesions during full heparinization for cardiopulmonary bypass or after mechanical valve replacement is rare but feared because it increases the risk of devastating intracranial hemorrhage.

Cerebrovascular manifestations of AIE often cause the first symptoms; that is, transient or persistent hemiparesis

\footnotetext{
From the Department of Cardiac Surgery, Heart Center Leipzig, University of Leipzig, Leipzig, Germany.

Disclosures: Authors have nothing to disclose with regard to commercial support.

M.M. and F.G. contributed equally to this study.

Read at the 93rd Annual Meeting of The American Association for Thoracic Surgery, Minneapolis, Minnesota, May 4-8, 2013.

Received for publication May 6, 2013; revisions received Sept 10, 2013; accepted for publication Oct 13, 2013.

Address for reprints: Martin Misfeld, MD, PhD, Department of Cardiac Surgery, Heart Center Leipzig, University of Leipzig, Struempellstrasse 39, D-04289 Leipzig, Germany (E-mail: martinmisfeld@yahoo.de). 0022-5223/\$36.00

Copyright (C) 2014 Published by Elsevier Inc. on behalf of The American Association for Thoracic Surgery

http://dx.doi.org/10.1016/j.jtcvs.2013.10.076
}

or hemiplegia or even unspecific headache, meningitis, altered level of consciousness, or severe seizures. According to the current practice guidelines for the management of AIE, ${ }^{3,4}$ preoperative cerebral imaging should be obtained to rule out septic embolic lesions, which carry a high risk for hemorrhagic transformation. However, asymptomatic so-called silent cerebral embolism has been previously described and its influence on short- and longterm outcome compared with symptomatic embolism is controversial. Asymptomatic lesions may be missed if routine preoperative computed tomography (CT) scans are not obtained, and fatal neurologic injury due to hemorrhagic transformation may cause excessive early mortality. Moreover, risk factors identifying patients who are at the highest risk for cerebral embolism may pose an additional decision: should CT scans should be obtained even in asymptomatic patients? ? $^{5}$

With our study we intended to determine the incidence of cerebral embolism in cardiac surgical patients with AIE and to investigate the predictors of unfavorable short- and longterm outcome after surgery for infective endocarditis complicated by cerebral embolism. 


\section{Abbreviations and Acronyms}

AIE $=$ acute infective endocarditis

$\mathrm{CT}=$ computed tomography

\section{PATIENTS AND METHODS}

A review of our institutional database identified 1571 consecutive patients who underwent operation for infective endocarditis from May 1995 through March 2012. Clinical data were derived from our institutional database and patient records. The local ethics committee did not require additional individual patient consent.

Infective endocarditis was diagnosed by the modified Duke criteria ${ }^{6}$ and vegetations were classified by transthoracic or transesophageal echocardiography. Vegetations were measured in a minimum of 2 planes and the maximum length of the vegetations was used. Blood cultures were routinely taken on admission and at the first postoperative day. Routine preoperative cranial, thoracic, and abdominal CT scans were performed in all patients admitted with the diagnosis of AIE to preoperatively rule out septic embolic lesions. Overall, 375 patients $(24 \%)$ presented with confirmed cerebral embolism. Mean age was $61.8 \pm 13.6$ years. Two hundred sixtysix patients were men $(70.9 \%)$. A detailed summary of the clinical data is presented in Table 1.

\section{Antibacterial Therapy}

In all patients, empiric antibiotic treatment was immediately initiated after diagnosis. A preoperative antibiotic therapy was initiated in 1260 out of 1571 patients $(80 \%)$ and in 338 out of 375 patients $(89 \%)$ with cerebral embolism. In the remaining patients antibiotic treatment was initiated immediately after surgery according to antibiotic resistance testing.

\section{Operative Methods}

According to current guidelines, indications for surgical intervention are large vegetations $\geq 10 \mathrm{~mm}(\mathrm{n}=261 ; 69.6 \%)$, prosthetic valve endocarditis ( $\mathrm{n}=90 ; 24 \%)$, abscess formation, fungal infection, Staphylococcus aureus endocarditis $(\mathrm{n}=143 ; 38.1 \%)$, congestive heart failure $(\mathrm{n}=53$; $14.1 \%)$, or failure of medical treatment.

In patients requiring aortic valve replacement, most patients received either a stented bioprosthetic valve $(\mathrm{n}=141)$ or a stentless bioprosthetic valve $(\mathrm{n}=30)$. A homograft was used in 11 patients, and 11 patients received a mechanical valve. In 7 patients, a reconstruction of the native valve was possible $(3.8 \%)$.

Among all patients with cerebral embolism, urgent mitral valve surgery was required in 222 out of 375 patients $(59.2 \%)$ and the patients received mostly valve replacement with a biological $(n=134)$ or a mechanical $(n=60)$ valve. In 15 patients, the mitral valve was amenable for repair. Concomitant repair of the tricuspid aortic valve was necessary in 22 patients, whereas 9 patients required tricuspid valve replacement. Other concomitant procedures were coronary artery bypass grafting in 66 patients $(18 \%)$, replacement of the ascending aorta in 17 patients $(5 \%)$, and partial arch replacement in 3 patients ( $1 \%$ ). Further operative details are listed in Table 2.

\section{Follow-up}

Our institutional database is continuously and prospectively maintained by research personnel. Follow-up data for long-term survival or adverse

TABLE 1. Baseline patient characteristics

\begin{tabular}{|c|c|c|c|c|}
\hline Variable & All patients $(n=1571)$ & Cerebral embolism $(n=375)$ & No cerebral embolism $(n=1196)$ & $P$ value \\
\hline Age, y & $61.6 \pm 14.8$ & $61.8 \pm 13.6(12-88)^{*}$ & $61.5 \pm 15.2$ & .78 \\
\hline Female, $\%$ & $477(30)$ & $109(29)$ & $368(31)$ & .56 \\
\hline LVEF, \% & $56.1 \pm 12.6$ & $56.7 \pm 12.0$ & $55.9 \pm 12.7$ & .44 \\
\hline \multicolumn{5}{|l|}{ Risk factors } \\
\hline Hypertension & $971(62)$ & $233(62)$ & $738(62)$ & .95 \\
\hline History of smoking & 445 (28) & 109 (29) & $336(28)$ & .74 \\
\hline Diabetes & $519(33)$ & $128(34)$ & $391(33)$ & .62 \\
\hline COPD & $184(12)$ & $40(11)$ & $144(12)$ & .52 \\
\hline Hyperlipidemia & $430(27)$ & $87(23)$ & $343(29)$ & .04 \\
\hline Preoperative dialysis & $109(7)$ & $25(7)$ & $84(7)$ & .91 \\
\hline LCO preoperative & $170(11)$ & $53(14)$ & $117(10)$ & .02 \\
\hline Logistic EuroSCORE, mean & $36.2 \pm 25.8$ & $43.1 \pm 25.4$ & $34.0 \pm 25.6$ & $<.01$ \\
\hline \multicolumn{5}{|l|}{ Affected valve } \\
\hline Single aortic & $732(47)$ & $165(44)$ & $567(47)$ & .26 \\
\hline Single mitral & $487(31)$ & $132(36)$ & $355(30)$ & .05 \\
\hline Aortic and mitral & $223(15)$ & 64 (17) & $159(13)$ & .07 \\
\hline Kissing mitral $\dagger$ & $201(13)$ & $51(14)$ & $150(13)$ & .60 \\
\hline Tricuspid valve & $108(7)$ & $12(3)$ & $96(8)$ & $<.01$ \\
\hline Pulmonary valve & $24(2)$ & $2(1)$ & $22(2)$ & .09 \\
\hline \multicolumn{5}{|l|}{ Vegetation size, $\mathrm{mm}$} \\
\hline$<5$ & $300(19)$ & $37(10)$ & $263(22)$ & $<.01$ \\
\hline$\geq 5$ to $<10$ & $276(18)$ & $72(19)$ & 204 (17) & .35 \\
\hline$\geq 10$ & $940(60)$ & $261(70)$ & $679(57)$ & $<.01$ \\
\hline N/A & 55 & 5 & 50 & - \\
\hline Prosthetic valve endocarditis & $383(24)$ & $90(24)$ & $293(25)$ & .89 \\
\hline Previous cardiac surgery & $435(28)$ & $102(27)$ & $333(28)$ & .84 \\
\hline
\end{tabular}

Values are presented as mean $\pm \mathrm{SD}$ or $\mathrm{n}(\%) . L V E F$, Left ventricular ejection fraction; $C O P D$, chronic obstructive pulmonary disease; $L C O$, low cardiac output syndrome; $N / A$, not applicable. *Range. $\dagger$ Endocarditis affecting the anterior leaflet of the mitral valve as well as the aortic valve. 
TABLE 2. Intraoperative data

\begin{tabular}{|c|c|c|c|c|}
\hline & All patients $(n=1571)$ & Cerebral embolism $(n=375)$ & No cerebral embolism $(n=1196)$ & $P$ value \\
\hline CPB time, $\min$ & $129 \pm 71$ & $128 \pm 76$ & $129 \pm 69$ & .35 \\
\hline Minimal temp, ${ }^{\circ} \mathrm{C}$ & $32.6 \pm 2.3$ & $32.8 \pm 2.3$ & $32.5 \pm 2.3$ & $<.01$ \\
\hline \multicolumn{5}{|l|}{ Procedures } \\
\hline Aortic valve replacement & $1017(65)$ & $239(64)$ & $778(65)$ & .66 \\
\hline Aortic valve repair & $19(1)$ & $7(2)$ & $12(1)$ & .18 \\
\hline Mitral valve replacement & $705(45)$ & $194(52)$ & $511(43)$ & $<.01$ \\
\hline Mitral valve repair & $113(7)$ & $15(4)$ & $98(8)$ & $<.01$ \\
\hline Tricuspid valve replacement & $40(3)$ & $3(1)$ & $37(3)$ & .01 \\
\hline Tricuspid valve repair & $118(8)$ & $22(6)$ & $96(8)$ & .06 \\
\hline \multicolumn{5}{|l|}{ Concomitant procedures } \\
\hline CABG & $260(17)$ & $66(18)$ & $194(16)$ & .23 \\
\hline Replacement of the ascending aorta & $74(5)$ & $17(5)$ & $57(5)$ & 1.0 \\
\hline Partial arch replacement & $15(1)$ & $3(1)$ & $12(1)$ & 1.0 \\
\hline
\end{tabular}

Values are presented as mean \pm standard deviation or $\mathrm{n}(\%) . C P B$, Cardiopulmonary bypass; $C A B G$, coronary artery bypass grafting.

outcomes are routinely gained either by direct telephone interview with the patient, a close relative, or the referring physician.

\section{Statistical Methods}

Characteristics are described as means \pm standard deviations. Continuous data were analyzed using the Wilcoxon log-rank test or MannWhitney $U$ test. Long-time survival analysis was performed by calculating Kaplan-Meier life tables. Groups were compared using the log-rank test. Categorical data were analyzed using $\chi^{2}$ or Fisher exact test. All tests were performed 2-sided. Risk factors for cerebral embolism and hospital mortality were identified using a multivariable binary regression analysis. The linearized mortality rate was calculated as 100 times the total number of deaths divided by the total person-years of follow-up. Follow-up time for survival was measured from the date of the operation to either the date of death or the date of the last alive contact. The Cox proportional hazards model was used to examine the risk factors for long-term death. The assumptions of the proportional hazards model were checked graphically. Univariable risk factors with a $P$ value $<.1$ were included in the multivariable analysis.

\section{RESULTS}

Overall mean follow-up time of the entire cohort of patients admitted with AIE and cerebral embolism $(\mathrm{N}=375)$ was $3.1 \pm 3.8$ years, with a maximum followup time of 16.9 years. An isolated left-sided endocarditis was present in 362 patients $(96.5 \%)$, whereas $3.5 \%$ had either right-sided endocarditis or combined left- and rightsided endocarditis. Ninety patients had prosthetic valve endocarditis $(24 \%)$. One hundred sixty-five patients $(44 \%)$ had single aortic valve endocarditis, whereas 133 patients $(35.5 \%)$ had single mitral valve endocarditis. Combined affliction of the aortic and the mitral valve was present in 64 patients $(17.1 \%)$. One hundred two patients had previously undergone cardiac surgery $(27.2 \%)$. About $23.5 \%$ of patients with prosthetic valve endocarditis had cerebral embolism; $37.8 \%$ of those were asymptomatic.

\section{Causative Pathogens}

In most patients, $S$ aureus was the causative pathogen for AIE $(n=143 ; 38.1 \%)$, followed by streptococci in 78 patients $(21 \%)$ and Enterococcus faecalis in 49 patients $(13 \%)$. Staphylococcus epidermidis was the pathogen in 25 patients $(7 \%)$. Other bacteria or fungi were present in 38 patients $(10 \%)$. In 42 of 375 patients, the preoperative blood cultures were sterile (11\%). S aureus was significantly associated with an impaired prognosis compared with patients with other pathogens: cumulative survival was $49 \% \pm 4 \%$ versus $63 \% \pm 3 \%$ after 1 year $(P=.04)$ and $38 \% \pm 5 \%$ versus $51 \% \pm 4 \%$ at 5 years, respectively $(P<.01)$.

\section{Preoperative Symptomatology}

Two hundred forty out of 375 patients (64\%) had symptomatic cerebral embolism, whereas hemiparesis or hemiplegia was present in 110 patients. An altered level of consciousness was detected in 60 patients $(16 \%), 8$ patients experienced seizures $(2 \%)$, and 11 patients had symptoms who were characteristic of meningitis or septic encephalitis. Five patients experienced intracerebral bleeding $(1 \%)$.

\section{Risk Factors for Cerebral Embolism in the Study Cohort}

A multivariate regression analysis identified a vegetation size exceeding $10 \mathrm{~mm}$ (odds ratio [OR], 1.40; 95\% confidence interval $[\mathrm{CI}], 1.07-1.82 ; P=.01)$, affliction of the aortic valve (OR, $2.43 ; 95 \% \mathrm{CI}, 1.58-3.72 ; P<.01)$ or the mitral valve (OR, 2.76; 95\% CI, 1.82-4.20; $P<.01)$, preoperative antibiotic therapy (OR, $1.91 ; 95 \% \mathrm{CI}$, $1.27-2.87 ; P<.01$ ), and $S$ aureus (OR, $1.89 ; 95 \% \mathrm{CI}$, $1.43-2.48 ; P<.01)$ as significant risk factors for cerebral embolism. Age and sex were not found to be associated with a higher risk of cerebral embolism. In patients with vegetations $<5 \mathrm{~mm}$, the rate of cerebral embolism was $12.3 \%(\mathrm{n}=37$ out of 300$)$ compared with $27.8 \%$ in patients with vegetations $\geq 10 \mathrm{~mm}(\mathrm{n}=261$ out of 940 ; $P<.01)$. The rate of cerebral embolism in patients with a positive blood culture result was almost twice as high as 
TABLE 3. Reasons for hospital death

\begin{tabular}{lc}
\hline \multicolumn{1}{c}{ Reason for hospital death } & No. of patients $(\mathbf{N}=\mathbf{8 8})$ \\
\hline Septic shock with multiorgan failure & $32(40)$ \\
Cerebral complications & $12(15)$ \\
Cardiac* & $18(22)$ \\
Gastrointestinal & $7(9)$ \\
Hemorrhagic shock & $4(5)$ \\
Pulmonary complications & $2(2)$ \\
Other & $6(7)$ \\
\hline
\end{tabular}

Values are presented as $\mathrm{n}(\%)$. * Low cardiac output syndrome.

in patients with negative blood culture results $(26.4 \%$ [ $\mathrm{n}=333$ out of 1263$]$ vs $13.8 \%[\mathrm{n}=42$ out of 305$]$; $P<.01)$.

\section{Pattern of Embolic Lesions}

The incidence of further septic embolic lesions was significantly higher in patients with cerebral embolism; that is, almost half of patients with cerebral embolism also experienced septic embolic lesions to the spleen (186 out of $375 ; 49.1 \%$ ) compared with an incidence of $22.1 \%$ in patients without cerebral embolism (294 out of 1196 ; $P<.01$ ). Renal lesions were detected in $24.3 \%$ compared with $7.0 \%$ (91 out of 375 vs 84 out of 1196 ; $P<.01)$, whereas pulmonary lesions were less frequent in patients with cerebral embolism ( 7 out of 375 vs 64 out of $1196 ; 1.9 \%$ vs $5.4 \% ; P=.04)$.

\section{Hospital Mortality and Short-Term Outcome}

Hospital mortality, defined as death within 30 days or inhospital death, was $21.6 \%(\mathrm{n}=81$ patients $)$, which was not inferior to patients without cerebral embolism (hospital mortality, $19.7 \% ; P=.51$ ). Main reason for hospital death was septic shock with consecutive multiorgan failure in $40 \%(\mathrm{n}=32)$, followed by low cardiac output syndrome in $22 \%(\mathrm{n}=18$ patients). A total of $15 \%$ of patients died from cerebral complications $(\mathrm{n}=12)$; most from cerebral edema (Table 3).

Risk factors identified for hospital mortality among all patients with AIE ( $\mathrm{n}=81$ early deaths) by multivariable binary regression analysis were female gender $(\mathrm{OR}, 1.6 ; 95 \%$ CI, $1.2-2.1 ; P=.01)$, age at surgery (OR, $1.03 ; 95 \% \mathrm{CI}$, $1.02-1.05 ; P<.01)$, low cardiac output syndrome (OR, $2.5 ; 95 \% \mathrm{CI}, 1.7-3.6 ; P<.01)$, renal insufficiency requiring dialysis (OR, 3.3; 95\% CI, 2.2-5.1; $P<.01$ ), and impaired left ventricular ejection fraction (OR, 1.3; 95\% CI, 1.2-1.5; $P<.01)$. Cerebral embolism did not appear as a risk factor for early mortality (OR, $1.1 ; 95 \% \mathrm{CI}, 0.8-1.5 ; P=.51)$.

\section{Postoperative Neurologic Outcome}

Postoperative neurologic outcome in patients with silent versus symptomatic embolism was not influenced by preoperative symptomatology: 30 patients had postoperative hemiparesis or hemiplegia (silent vs symptomatic

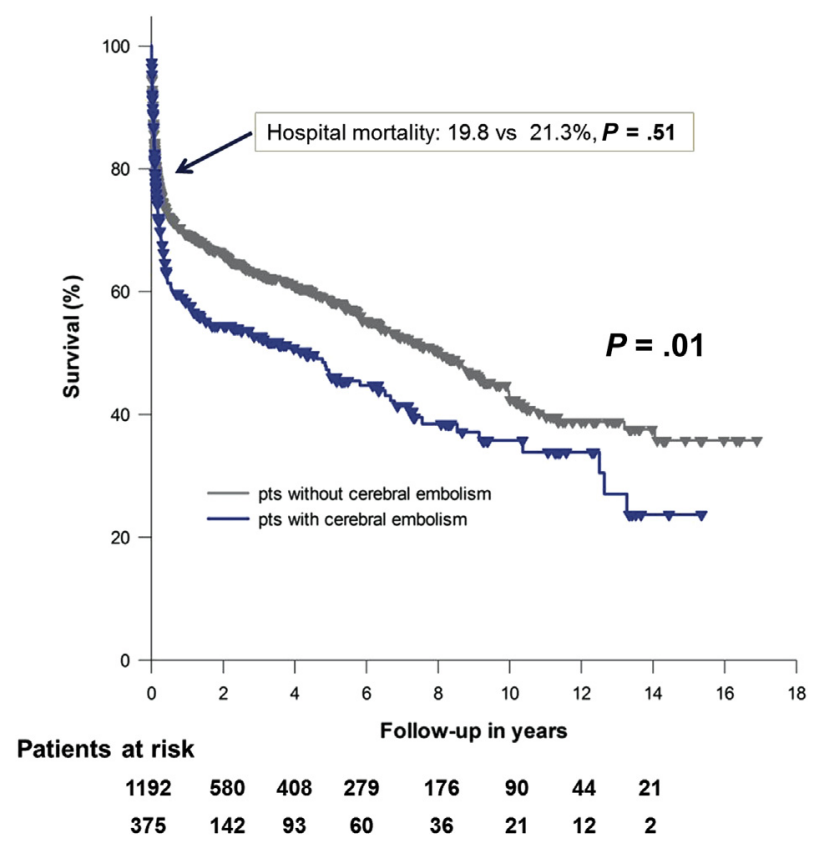

FIGURE 1. Long-term survival in patients with cerebral embolism compared with patients without cerebral embolism. pts, Patients.

embolism, 18 vs 12 patients; $P=.69$ ). Seizures were observed in 39 patients ( 15 vs 24 patients; $P=.73$ ). A total of 7 patients had severe intracerebral bleeding $(2 \% ; 3$ vs 4 patients; $P=.71$ ). From these 7 patients, 3 died within 30 days after the operation, with 1 receiving acute craniotomy for decompression. Another patient needed decompressive craniotomy due to cerebral edema. Three patients were managed medically, with 1 patient experiencing permanent hemiparesis; 1 patient presented with recurrent epileptic seizures and was treated medically.

\section{Long-Term Survival and Risk Factors for Long-Term Death}

Long-term survival was significantly influenced by cerebral embolism. Patients with preoperative cerebral embolism had a 5-year survival of $46 \% \pm 3 \%$ compared with $57 \% \pm 2 \%$ in patients without cerebral embolism $(P<.01)$. Long-term survival after 10 years was $42 \% \pm$ $2 \%$ versus $35 \% \pm 4 \%$, respectively $(P<.01)$ (see Figure 1).

However, long-term survival was not influenced by preoperative symptomatology, but by the sole presence of cerebral embolism: patients with silent cerebral embolism had an equally reduced short- and long-term mortality compared with patients with preoperative neurologic symptoms. There were no significant differences comparing short-term survival after 1 year $(56 \% \pm 5 \%$ vs $59 \% \pm$ $3 \%$ for silent vs symptomatic embolism; $P=.77$ ) and long-term survival after 5 years $(45 \% \pm 5 \%$ vs $47 \% \pm$ $4 \%$ for silent vs symptomatic embolism; $P=.83$ ) and 


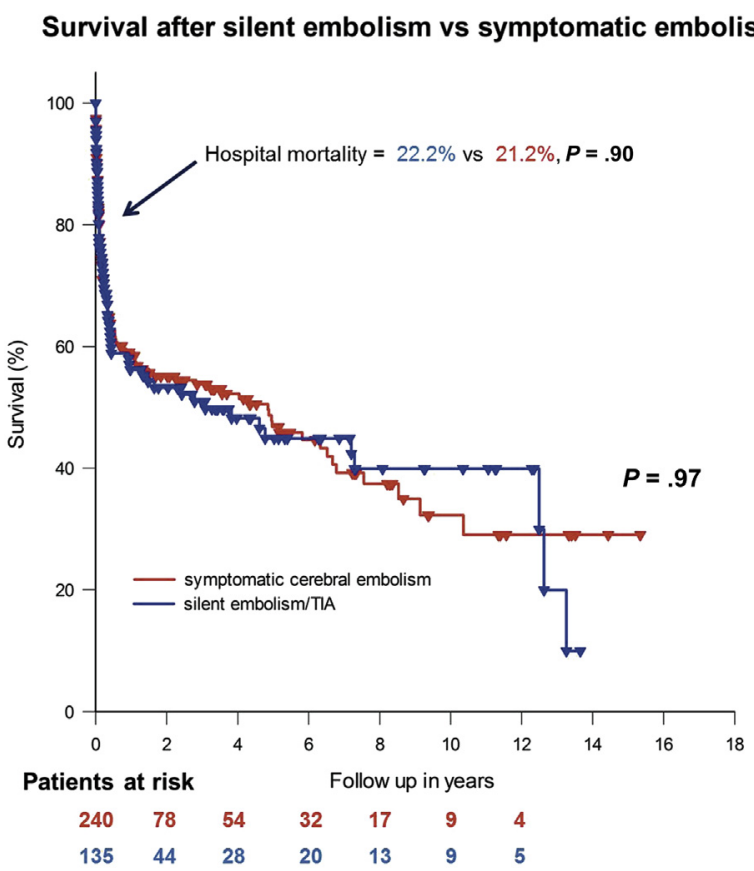

FIGURE 2. Long-term survival in patients with silent cerebral embolism or transient ischemic attack (TIA) (blue line) compared with patients symptomatic cerebral embolism (red line).

10 years $(40 \% \pm 6 \%$ vs $32 \% \pm 5 \%$ for silent vs symptomatic embolism; $P=.86$ ) (see also Figure 2). Risk factors for long-term death in patients with cerebral embolism were cardiopulmonary bypass time $(P<.01)$, preoperative dialysis $(P<.01)$, low cardiac output syndrome $(P=.01)$, age at surgery $(P<.01)$, and chronic obstructive pulmonary disease $(P=.03)$.

Considering all patients with cerebral embolism receiving either biological or mechanical valve replacement, survival was not statistically different between patients with mechanical valve $(\mathrm{n}=94)$ replacement compared with biological valve replacement $(\mathrm{n}=266)$, with a cumulative 10 -year survival of $22 \% \pm 7 \%$ versus $43 \% \pm 6 \%(P=.77)$. However, excluding patients who died within 30 days or before hospital discharge, patients receiving a mechanical valve ( $\mathrm{n}=74$ vs $\mathrm{n}=206$ biological valves) clearly had a favorable long-term survival $(29 \% \pm$ $9 \%$ vs $55 \% \pm 7 \%$ after 10 years, mechanical vs biological valve replacement; $P=.04$ ), but patients receiving a mechanical valve were also significantly younger than their peers who received a bioprosthetic valve $(65.4 \pm 11.9$ vs $53.7 \pm 12.6$ years; $P<.01$ ).

In patients receiving aortic valve replacement only, there was no difference in cumulative survival between patients with a biological valve versus patients with a mechanical valve $(34 \% \pm 8 \%$ vs $49 \% \pm 10 \%$ after 10 years; $P=.34$; excluding hospital mortality, $42 \% \pm 10 \%$ vs $59 \% \pm 12 \%$ after 10 years; $P=.27$ ).

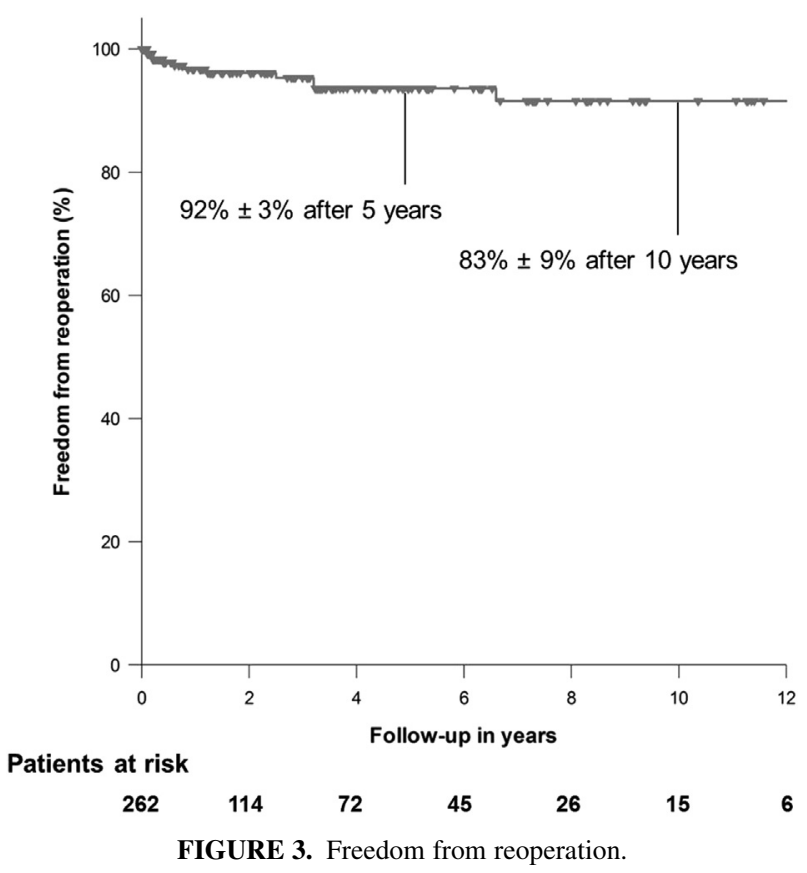

\section{Freedom From Reoperation}

Freedom from reoperation in patients with septic cerebral embolism was $92 \% \pm 3 \%$ after 5 years and $83 \% \pm 9 \%$ after a follow-up period of 10 years (see Figure 3). A total of 13 patients needed reoperation due to prosthetic valve endocarditis at a mean of $2.08 \pm 3.65$ years (median, 5 months).

\section{DISCUSSION}

Cerebrovascular complications of infective endocarditis are frequent. ${ }^{1}$ The rate of cerebrovascular complications in our study cohort was $24 \%$ (375 out of 1571 patients), which is consistent with previously published studies ${ }^{7,8}$ : Thuny and colleagues ${ }^{2}$ found a rate of cerebrovascular complications of $22.2 \%$ in their cohort of 496 patients, with no difference in prosthetic versus native valve endocarditis. They also found that cerebrovascular complications were more frequent in patients with mitral valve endocarditis, which is also consistent with our results because mitral valve endocarditis had the highest OR for cerebral embolism (OR, $2.76 ; 95 \% \mathrm{CI}, 1.82-4.20 ; P<.01)$. In concordance with previously published studies, the risk for cerebral embolism was higher in patients with mitral valve endocarditis than aortic valve endocarditis (OR, 2.43; 95\% CI, 1.58-3.72). ${ }^{9}$ The rate of symptomatic cerebral embolism was $64 \%$ ( 240 out of 375 patients) in our institutional cohort.

In our study cohort, risk factors for cerebral embolism were endocarditis of the mitral valve and/or the aortic valve, vegetation size $>10 \mathrm{~mm}$, preoperatively initiated empiric antibiotic therapy, and $S$ aureus. Various risk factors we found have been previously described. In 2004, Deprèle and colleagues ${ }^{10}$ and Thuny and colleagues ${ }^{11}$ also found 
that vegetations $>10 \mathrm{~mm}$ in size were significantly associated with systemic embolism and Di Silva and colleagues ${ }^{12}$ underlined that large vegetations $>15 \mathrm{~mm}$ predict an even higher risk of embolization. In addition, Heiro and colleagues $^{7}$ and Corral and colleagues ${ }^{8}$ found that $S$ aureus is significantly associated with cerebral complications in infective endocarditis.

Our study cohort shows a clear predominance of biological valves compared with mechanical valves. In past years, the implantation of biological valves in patients with AIE has been preferred according to our institutional policy. Commonly, biological valves are preferred if cerebral septic embolic lesions are present because anticoagulation regimens do not have to be as strict as in mechanical heart valves, in particular if hemorrhagic transformation of a cerebral infarction is observed postoperatively. Generally, patients with biological heart valves are administered aspirin $100 \mathrm{mg}$ daily and intravenous heparin (partial thromboplastin time, 60-80 seconds) until they are transferred to the general ward and unless there are relevant bleeding complications. Afterward, recipients of biological valves are administered phenprocoumon for 2 to 3 months in the absence of contraindications. Mechanical heart valves are generally treated with the anticoagulant intravenous heparin (partial thromboplastin time 60-80 seconds) until transferred to the general ward and followed with lifelong anticoagulation therapy.

Interestingly, prosthetic valve endocarditis was not associated with a higher incidence of cerebral embolism compared with native valve endocarditis (native valve endocarditis, $24.1 \%$ vs prosthetic valve endocarditis, $23.5 \%$; $P=.89$ ). These findings correspond to Delahaye and colleagues ${ }^{13}$ who also found no difference in the rate of cerebrovascular events in native versus prosthetic valve endocarditis.

Furthermore, it was previously hypothesized that older patients might have a higher risk for systemic embolism due to preexisting vascular disease. ${ }^{5}$ Interestingly, patients with septic cerebral embolism were not significantly older than patients without cerebral embolism in our study $(61.8 \pm 13.6$ vs $61.5 \pm 15.2$ years; $P=.78)$.

Moreover, we found that preoperative antibiotic treatment was a significant risk factor for cerebral embolism in our cohort (OR, 1.91; 95\% CI, 1.27-2.87; $P<.01)$. A possible explanation is that preoperative antibiotic therapy in patients with left-sided endocarditis and large vegetations may lead to a fragmentation of the vegetations and consecutively lead to a higher incidence of systemic embolism. Unfortunately, it was not possible to retrospectively obtain reliable data about the exact duration of preoperative antibiotic therapy.

In 2002 Vilacosta and colleagues ${ }^{9}$ found $65 \%$ of embolic events that occurred during antibiotic therapy were detected during the first 2 weeks after initiation of the antimicrobial treatment-a striking finding that has to be verified by future prospective studies, because it is a clue that the dogmatic principle of "hit hard and early" in the antibiotic therapy of AIE with large vegetations might be open for debate and puts more emphasis on early surgical therapy. However, among patients who had not received preoperative empiric intravenous antibiotic therapy, the proportion of patients with vegetations $>10 \mathrm{~mm}$ was significantly lower (86 out of 311 patients vs 853 out of 1260 patients; $P<.01$ ), indicating that there might be a higher proportion of subacute or subsided endocarditis in this group, who were in part intraoperatively diagnosed as having endocarditis.

Cerebral embolism was significantly associated with a higher incidence of lesions to the spleen and the kidneys, whereas pulmonary lesions were much less frequent. Apart from the fact that tricuspid valve endocarditis has a higher incidence of pulmonary embolism, these findings suggest that cerebral embolism is often an expression of a generally septic course of endocarditis. Given the fact that most patients with cerebral lesions died from sepsis with multiorgan failure, sepsis seems to be more threatening to patients than hemorrhagic transformation of a septic embolic cerebral stroke.

Hospital mortality in our high-risk cohort was $22 \%$-still high, but comparable to previously published studies. The main reason for perioperative death was septic shock with consecutive multiorgan failure. Generally, the occurrence of sepsis hemodynamics seems to be a main predictor for short- and long-term outcome. In a previously published study from Ruttmann and colleagues, ${ }^{14}$ sepsis was the main reason for hospital death. Hill and colleagues ${ }^{15}$ also found septic shock and $S$ aureus infection were significantly associated with 6-month mortality. These findings emphasize the importance of preventing septic dissemination of the vegetations, either by early operative treatment and/or by early and aggressive antimicrobial treatment.

We observed only 7 patients ( $2 \%)$ with severe postoperative intracerebral bleeding with only 2 patients dying within the first 30 days, which is a remarkably low rate and much lower than we expected. However, Ruttmann and colleagues ${ }^{14}$ also found only 1 of 65 patients with septic cerebral embolism dying from postoperative intracerebral hemorrhage, so the risk of fatal perioperative cerebral bleeding as a result of anticoagulation during cardiopulmonary bypass might be commonly overestimated. Yoshioka and colleagues ${ }^{16}$ reported only 1 silent hemorrhagic transformation of a cerebral septic embolic infarction in their study cohort of 64 patients. Various reasons for the beneficial neurologic outcome after septic embolic stroke have been previously discussed: Ruttmann and colleagues ${ }^{14}$ hypothesized that anticoagulation during cardiopulmonary bypass might lead to fragmentation and a more peripheral dislocation of the embolus, explaining a relatively good neurologic outcome. They argue that patients with septic 
embolism are commonly younger and have a better vascular status than the typical patient experiencing a stroke. However in patients with infective endocarditis, cerebral microbleeding detected by magnetic resonance scans are thought to be indicators of poor vascular status and therefore might be predictive for intracranial hemorrhage. ${ }^{17}$ Yoshioka and colleagues ${ }^{16}$ stated that reperfusion injury is less likely to occur because bacterial embolism was unlikely to be lysed due to a specific thrombus composition.

Despite improvements in diagnostics and therapy, patients with AIE still have a poor long-term prognosis, which is even inferior in patients with septic cerebral embolism. In our study, patients with cerebral lesions showed a 5-year survival of $46 \% \pm 3 \%$ compared with $57 \% \pm 2 \%$ in patients without cerebral embolism $(P<.01)$, with a 10 -year survival after 10 years of $42 \% \pm 2 \%$ versus $35 \% \pm 4 \%$, respectively $(P<.01)$. However, we did not find a significant difference with regard to long-term survival in patients with preoperative symptomatic versus silent embolism. In contrast, Thuny and colleagues ${ }^{2}$ found a significantly better long-term survival in patients with silent embolism or transient ischemic attack compared with symptomatic embolism. This might be due to different diagnostic criteria. Our definition criteria of symptomatic embolism also included patients with unspecific symptoms such as an altered level of consciousness or headache because these might be clinical decision criteria to rule out septic embolism by CTor magnetic resonance imaging. In a prospective study from Chu and colleagues, ${ }^{18}$ septic embolism was an important predictor of early mortality, but the rate of patients receiving surgery for infective endocarditis was only $27 \%$.

Risk factors for long-term death in patients with cerebral embolism were cardiopulmonary bypass time $(P<.01)$, preoperative dialysis $(P<.01)$, low cardiac output syndrome $(P=.01)$, age at surgery $(P<.01)$, and chronic obstructive pulmonary disease $(P=.03)$.

These risk factors reflect common risk factors for mortality in patients undergoing cardiac surgery. Most of them are patient-related and not modifiable; that is, cannot be influenced by the surgeon. Even cardiopulmonary bypass time is to be considered as an indicator for the complexity of the required operation (eg, complicated by intraoperative findings, excessive tissue destruction, fragile tissue, and abscess formation). However, we believe that low cardiac output syndrome is either a sign of septic cardiomyopathy or excessive valve destruction leading to significant valve regurgitation, which indicates that these patients would probably have benefited from earlier referral to a cardiac surgeon.

Our data do not support the notion that delaying or avoiding surgery in high-risk patients results in better survival.

\section{Limitations}

The results of our study may be subject to various selection biases, such as patients with large cerebral infarction or severe preoperative intracranial hemorrhage detected by preoperative routine $\mathrm{CT}$ scans were considered not suitable for surgery and were possibly not referred to our hospital during the acute phase. Furthermore, the time from onset of the symptoms until surgery may pose a confounding variable that could not be sufficiently detected during retrospective analysis. Due to this fact, it is not possible to determine the optimal timing for heart valve surgery after a cerebrovascular event has occurred because of septic embolism. However, this was not the subject of our study, but this important fact remains to be clarified. Several authors propose a safe interval for surgical therapy, which is either in the first 72 hours after onset of an embolic event or otherwise after a delay of 4 weeks. ${ }^{19-21}$

Septic cerebral embolism was generally ruled out by preoperative routine CT scans, not by magnetic resonance imaging, which might be more sensitive in the early phase of an acute ischemic stroke. Because it is known that ischemic cerebral lesions show a time latency of about 2 to 6 hours after they can be identified in cerebral CT scans, ${ }^{22}$ some septic embolic events may have been missed if the CT scans were obtained during the early phase. Furthermore, magnetic resonance imaging seems to be more sensitive in detecting cerebral embolism compared with CT studies, resulting in a far higher incidence of acute brain embolization in infective endocarditis than previously assumed. ${ }^{1,23,24}$ Moreover, we did not perform cerebrospinal fluid analyses for neurochemical markers of acute brain damage because lumbar punctures preceding full heparinization for cardiopulmonary bypass may pose a high risk for spinal hematoma with devastating consequences and their clinical relevance is doubtful. Moreover, because we are a tertiary reference center for cardiac surgery, our patients were not routinely seen by an experienced neurologist before the surgical intervention, so some patients might have been mistakenly assigned to the silent embolism group despite presenting with latent neurologic symptoms.

\section{CONCLUSIONS}

In patients with infective endocarditis, silent cerebral embolism can frequently be detected by routine preoperative $\mathrm{CT}$ scans and is a predictor of an unfavorable short- and long-term outcome. Because long-term survival in patients with cerebral embolism is independent from actual symptomatology, routine preoperative CT or magnetic resonance scans are necessary to detect silent embolism. Particularly in patients with a vegetation sized $>10 \mathrm{~mm}$ and blood cultures positive for $S$ aureus, cerebral CT scans are mandatory to rule out septic cerebral embolism.

Patients with either symptomatic or asymptomatic embolism have to be closely monitored to early detect hemorrhagic transformation and consecutively adjust anticoagulation 
regimens. We also recommend neurologic consultation in all patients with cerebral embolism, in particular if hemorrhagic transformation or intracranial bleeding has occurred.

The authors thank Meinhard Mende, $\mathrm{PhD}$, for providing support concerning statistical analysis.

\section{References}

1. Snygg-Martin U, Gustafsson L, Rosengren L, Alsiö A, Ackerholm P, Andersson R, et al. Cerebrovascular complications in patients with left-sided infective endocarditis are common: a prospective study using magnetic resonance imaging and neurochemical brain damage markers. Clin Infect Dis. 2008;47:23-30.

2. Thuny F, Avierinos JF, Tribouilloy C, Giorgi R, Casalta JP, Milandre L, et al. Impact of cerebrovascular complications on mortality and neurologic outcome during infective endocarditis: a prospective multicentre study. Eur Heart J. 2007;28:1155-61.

3. Habib G, Hoen B, Tornos P, Thuny F, Prendergast B, Vilacosta I, et al., ESC Committee for Practice Guidelines. Guidelines on the prevention, diagnosis, and treatment of infective endocarditis (new version 2009): the Task Force on the Prevention, Diagnosis, and Treatment of Infective Endocarditis of the European Society of Cardiology (ESC). Endorsed by the European Society of Clinical Microbiology and Infectious Diseases (ESCMID) and the International Society of Chemotherapy (ISC) for Infection and Cancer. Eur Heart J. 2009;30:2369-413.

4. Byrne JG, Rezai K, Sanchez JA, Bernstein RA, Okum E, Leacche M, et al. Surgical management of endocarditis: the society of thoracic surgeons clinical practice guideline. Ann Thorac Surg. 2011;91:2012-9.

5. Baddour LM, Bayer AS. Cerebrovascular complications in patients with leftsided infective endocarditis: out of site, out of mind. Clin Infect Dis. 2008;47: 31-2.

6. Li JS, Sexton DJ, Mick N, Nettles R, Fowler VG Jr, Ryan T, et al. Proposed modifications to the Duke criteria for the diagnosis of infective endocarditis. Clin Infect Dis. 2000;30:633-8.

7. Heiro M, Nikoskelainen J, Engblom E, Kotilainen E, Marttila R, Kotilainen P. Neurologic manifestations of infective endocarditis: a 17-year experience in a teaching hospital in Finland. Arch Intern Med. 2000;160:2781-7.

8. Corral I, Martín-Dávila P, Fortún J, Navas E, Centella T, Moya JL, et al. Trends in neurological complications of endocarditis. J Neurol. 2007;254:1253-9.

9. Vilacosta I, Graupner C, San Román JA, Sarriá C, Ronderos R, Fernández C, et al. Risk of embolization after institution of antibiotic therapy for infective endocarditis. J Am Coll Cardiol. 2002;39:1489-95.

10. Deprèle C, Berthelot P, Lemetayer F, Comtet C, Fresard A, Cazorla C, et al. Risk factors for systemic emboli in infective endocarditis. Clin Microbiol Infect. 2004; 10:46-53.

11. Thuny F, Di Salvo G, Belliard O, Avierinos JF, Pergola V, Rosenberg V, et al. Risk of embolism and death in infective endocarditis: prognostic value of echocardiography: a prospective multicenter study. Circulation. 2005;112:69-75.

12. Di Salvo G, Habib G, Pergola V, Avierinos JF, Philip E, Casalta JP, et al. Echocardiography predicts embolic events in infective endocarditis. J Am Coll Cardiol. 2001;37:1069-76.

13. Delahaye JP, Poncet P, Malquarti V, Beaune J, Garé JP, Mann JM. Cerebrovascular accidents in infective endocarditis: role of anticoagulation. Eur Heart J. 1990; 11:1074-8.

14. Ruttmann E, Willeit J, Ulmer H, Chevtchik O, Höfer D, Poewe W, et al. Neurological outcome of septic cardioembolic stroke after infective endocarditis. Stroke. 2006;37:2094-9.

15. Hill EE, Herregods MC, Vanderschueren S, Claus P, Peetermans WE, Herijgers P. Outcome of patients requiring valve surgery during active infective endocarditis. Ann Thorac Surg. 2008;85:1564-9.

16. Yoshioka D, Sakaguchi T, Yamauchi T, Okazaki S, Miyagawa S, Nishi H. Impact of early surgical treatment on postoperative neurologic outcome for active infective endocarditis complicated by cerebral infarction. Ann Thorac Surg. 2012;94: 489-95.

17. Okazaki S, Sakaguchi M, Hyun B, Nagano K, Tagaya M, Sakata Y, et al. Cerebral microbleeds predict impending intracranial hemorrhage in infective endocarditis. Cerebrovasc Dis. 2011;32:483-8.

18. Chu VH, Cabell CH, Benjamin DK Jr, Kuniholm EF, Fowler VG Jr, Engemann J, et al. Early predictors of in-hospital death in infective endocarditis. Circulation. 2004;109:1745-9.
19. Derex L, Bonnefoy E, Delahaye F. Impact of stroke on therapeutic decision making in infective endocarditis. J Neurol. 2010;257:315-21.

20. Angstwurm K, Borges AC, Halle E, Schielke E, Einhäupl KM, Weber JR. Timing the valve replacement in infective endocarditis involving the brain. J Neurol. 2004;251:1220-6.

21. Piper C, Wiemer M, Schulte HD, Horstkotte D. Stroke is not a contraindication for urgent valve replacement in acute infective endocarditis. J Heart Valve Dis. 2001:10:703-11.

22. Von Kummer R, Allen KL, Holle R, Bozzao L, Bastianello S, Manelfe C, et al. Acute stroke: usefulness of early $\mathrm{CT}$ findings before thrombolytic therapy. Radiology. 1997;205:327-33.

23. Cooper HA, Thompson EC, Laureno R, Fuisz A, Mark AS, Lin M, et al. Subclinical brain embolization in left-sided infective endocarditis: results from the evaluation by MRI of the brains of patients with left-sided intracardiac solid masses (EMBOLISM) pilot study. Circulation. 2009;120:585-91.

24. Duval X, Iung B, Klein I, Brochet E, Thabut G, Arnoult F, et al., Resonance Magnetic Imaging at the Acute Phase of Endocarditis Study Group. Effect of early cerebral magnetic resonance imaging on clinical decisions in infective endocarditis: a prospective study. Ann Intern Med. 2010;152:497-504.

\section{Discussion}

Dr Marc Ruel (Ottawa, Canada). Dr Misfeld and colleagues have presented their findings in 375 patients who had surgery for acute infective endocarditis preoperatively complicated by cerebral embolism over an 18-year period. This subpopulation represents roughly a quarter of their operations for infective endocarditis. The work contains interesting descriptive information, some of which I will comment on.

When dealing with infective endocarditis there are essentially 2 questions: what technique to do and when to do it. We are getting much better at answering the first question. We have better antibiotics, better hemodynamic treatments, and we know that homografts are not mandatory, which was confirmed once more in your study where they were used relatively infrequently. And we also know that stroke is not a contraindication to surgery. Nevertheless, the perioperative mortality of these operations remains high, and in your study it was $22 \%$, which is not out of range but still high indeed.

When to intervene remains the big question. You have shown that 135 patients, representing roughly $9 \%$ of all your operated infective endocarditis patients, and $36 \%$ of the cerebral embolism subcohort, had asymptomatic cerebral embolism. One of your conclusions is that preoperative cerebral imaging is therefore of great importance. However, it remains to be determined to what extent cerebral imaging changes the management, provided that you do not find shift or a bleed on the computed tomography (CT) scan or magnetic resonance imaging scan.

There are other groups, such as the 1 from Osaka University presented last year, who found that perioperative hemorrhagic transformation of a stroke is relatively rare, and you have found this as well. But I believe that surgeons need to know if there are characteristics or predictors of what may lead to hemorrhagic transformation or cause significant brain edema with surgery.

There are groups, for instance, who have used an algorithm where surgery is delayed if a brain infarct with mass effect is found on head CT, or if there is an unruptured mycotic aneurysm present that would be clipped before surgery.

It is clear that more and more research points toward early surgical intervention in the case of infective endocarditis with cerebral embolization, on the basis that it does not increase mortality and may help prevent subsequent stroke. 
Here you make no claim, but you point out that preoperative antibiotic therapy was independently associated with cerebral embolism and, therefore, one should proceed to surgery quickly. Recent work from the International Collaboration on Endocarditis revealed a trend toward higher in-hospital mortality when surgery was performed within 7 days of the stroke at a $22 \%$ rate, versus only $12 \%$ when surgery was delayed to a later date.

There has to be some prognostic determinants related to the brain imaging, to the causative organism, to the status of the patient, to the valve itself, to the vegetations and their size, or to the hemodynamics that we just do not know and need to elucidate. Your research is a step in the right direction, but these many questions remain.

I have 2 questions. First, do you use the results of the head CT scan or magnetic resonance imaging scan as mere prognostic information for the patient and family, or are there some characteristics that are looked for to delay or perhaps in some cases expedite surgical intervention?

My second question is related to the first. Clearly not everyone who has a cerebral embolism and infective endocarditis has to undergo operation; for instance, in previous research only $23 \%$ did. Many patients are fine just with medical treatment. What are the patient or brain lesion characteristics that would entice you to operate right away versus waiting, perform another intervention such as neurosurgery first, or not operate at all. For instance, if a patient is stable with a smaller lesion but has infective endocarditis and cerebral embolism?

Dr Misfeld. Indication in Leipzig for performing surgery in this group of patients is always an individual decision and probably there are 4 aspects that are important.

First, the clinical presentation: What kind of neurologic complication is present? Does the patient have low cardiac output? Status of the other organ systems is important, too. Second, the therapeutic regimen at presentation: How many inotropes is the patient taking and what about the antibiotic regimen? For how long has the patient been taking antibiotics and what kind of bacteria have been cultured? Third, the echo images are most important, and it is probably not only the size of the vegetation, it is especially the characteristics of the vegetation; mobility, for example. Additional questions have to be answered: are there multiple vegetations, are both valves affected, and is an abscess present? This will all be part of the final decision, as well as the fourth aspect, the CT scan or magnetic resonance imaging findings. We routinely perform not only a CT scan of the brain, but also of the whole body.

With regard to the cranial CT scan we have some sort of insensitivity within the first time after a stroke where the lesions in the brain are probably not demarked and therefore not visible. You are also probably not able to detect microbleeds with the CT scan as you can do with magnetic resonance imaging. Microbleeds have been proposed to be a risk factor for later cerebral bleeding. However, CT scans are fast to perform and available everywhere.

I will give you an example. In a patient with a vegetation of $5 \mathrm{~mm}$ who has experienced a stroke, we would look at the kind of vegetation: Is it mobile? We would also look at the CT scan. If there is no lesion, but a clinical presentation of stroke, a surgeon is probably within this range of safety (before 72 hours) where the ischemic region has not been transformed with the potential risk of hemorrhage. In that patient, we would perform the surgery as soon as possible. Some even advocate that in a patient like this surgery may be protective against stroke by using a heart-lung-machine with hemodilation, hypothermia, and anticoagulation.

If that patient presents with a stroke and a lesion on the brain, it depends on the location and the size of the cerebral defect. It has been suggested that small-size lesions are probably safe, whatever "small" means, so probably 1 to $1.5 \mathrm{~cm}^{2}$ is a small lesion. The location of the lesion is also important. Is it more peripheral or central and are there multiple lesions or not?

It is also most important to consult a radiologist; a cardiologist; and, if there is a hemorrhagic transformation, the neurosurgeon. In the end it will be a team decision.

If there is a bigger lesion or multiple small lesions and already a shift in the brain's midline or an edema, you probably need to postpone the procedure. We would also postpone surgery in the presence of a hemorrhagic transformation.

At 1 stage you need to balance the increasing the risk of cerebral complications versus the risk of the operation itself. In these patients we watch and wait; we closely follow them clinically, with CT scans and echo. In some patients who had a hemorrhagic transformation and you postpone surgery, it ends up in an ethical question of if you operate or not.

Dr Antonio Laudito (Duluth, Minn). I have a question on the temporal pattern of embolization. Considering your experience and your review of the literature, are the patients embolizing in the first 48 hours or, as you say, 72 hours, and then the rate of embolization goes down? Could you confirm that kind of clinical scenario and temporal pattern of embolization?

Dr Misfeld. Usually we see the patients very late. We see patients who already have an abscess or they have embolized or they have experienced a stroke and then we see them after 1 or 2 weeks.

There is decreasing risk of stroke over time. If a patient has experienced a stroke once and is following a proper antibiotic treatment, probably the risk of re-stroke is less than assumed.

Dr Thierry Mesana (Ottawa, Canada). Do you have a particular recommendation for a prosthetic valve in these patients? How often would you put in a mechanical valve, and how do you manage anticoagulation in these patients after surgery?

Dr Misfeld. We follow the principle: Be radical and repair what you have to repair. The other principle is that we aim to drain the pus if there is an abscess into the mediastinum. So try to avoid patching abscesses.

It is not as important what kind of valve type you use. If there is a hemorrhagic transformation before the operation or a very high risk for that, we choose a biological valve-not a mechanical valve-to avoid anticoagulation. Age is not an issue for us with regard to valve choice. The patients have to survive the endocarditis.

We still use the homograft because we believe that without any artificial material, at least theoretically, this has some benefit with regard to the risk of reinfection. My advice is that you probably should stick to the valve type or the procedure you are used to. If you have never used a homograft before, you probably should not use a homograft in an endocarditis scenario with root abscess.

If the endocarditis only affects the cusps of the aortic valve, and there is no brain lesion or hemorrhage, then we would follow our normal regimen of putting in biological or mechanical valves. 
Dr Mesana. You do not exclude a mechanical valve in these patients?

Dr Misfeld. No, but our overall number of mechanical valves for younger patients without endocarditis is already very, very low.

Dr Harold Lazar (Boston, Mass). I have 1 final question. I wholeheartedly agree with you about running patients through a scanner when they come in with endocarditis. I have always been amazed to see other areas. How often have you seen other embolic sites associated with cerebral emboli? How often do you see it just as 1 area? In the presence of multiple emboli, multiple systems involved, the brain, spleen, usually kidney, would that make you want to operate earlier than if you just saw 1 isolated area?

Dr Misfeld. Excellent question. If there is no lesion of the brain but emboli are present in the spleen and kidney, for example, then you can assume that probably emboli are present in the brain that have not been detected yet by CT scan or they are too small. In more than one-third of cases we were able to find spleen embolization or abscesses and we had to take the spleen out in one-third of these patients. 